\title{
LA INVESTIGACIÓN SOBRE LA COMUNICACIÓN DE MASAS*
}

VV.AA.

Este memorándum constituye una declaración en favor de la investigación en la comunicación de masas, particularmente la prensa, la radio y el cine. Es el resultado de una serie de reuniones informales que se celebraron entre septiembre de 1939 y junio de 1940. El presente memorándum se redactó formalmente en una última reunión que se celebró en junio, en la cual participaron sus firmantes. Sin embargo, a lo largo del presente año, otras personas participaron en las discusiones, siendo los participantes más asiduos —aparte de los firmantes de este memorándum- R. J. Havighurst, Stacey May, I. A. Richards y David H. Stevens.

Las ideas expuestas en este memorándum, claro está, representan sólo las opiniones de aquellas personas que lo firman, y de ninguna manera pueden considerarse representativas de las organizaciones o instituciones a las que

* El texto de este documento - titulado originalmente «Research in Mass Communication. July 1940»— ha sido tomado del microfilm 59-1, que contiene documentos de la Fundación Rockefeller y se encuentra en el Mass Communication Research Center de la State Historical Society of Wiscounsin, en Madison (Wiscounsin). El documento es el último de una serie que se refiere a las conversaciones mantenidas en la ciudad de Nueva York, bajo los auspicios de la Fundación Rockefeller, sobre la investigación de la comunicación de masas, durante los años 1939 y 1940. Este texto, último del carrete de microfilm, viene precedido por breves textos de H. Lasswell, G. Gorer, I. A. Richards, D. Waples, L. Bryson, P. F. Lazarsfeld y R. S. Lynd, y un largo memorándum titulado "Public opinion and the emergency», fechado en diciembre de 1939, que alude a los comienzos de la guerra mundial en Europa.

\section{Reis}


están vinculadas esas personas. En el momento actual se está enviando el memorándum a un número de conocidos que se piensa que pueden tener interés en él, bien entendido que el memorándum es únicamente para la distribución privada y no puede, de ninguna manera, ser citado ni dado a la luz pública. Los autores agradecerían, por supuesto, cualesquiera comentarios o críticas que quisieran aportar los lectores del memorándum.

\author{
Lyman Bryson \\ Lloyd A. Free \\ Geoffrey Gorer \\ Harold D. Lasswell \\ Paul F. Lazarsfeld \\ Robert S. Lynd \\ John Marshall \\ Charles A, Siepmann \\ Donald Slesinger \\ Douglass Waples
}

Junio de 1940

Este informe es una declaración de una creencia, la creencia en el significado de tres hechos. Creemos, en primer lugar, que en los tiempos difíciles que nos esperan la opinión pública va a ser un factor decisivo. Si los Estados Unidos han de hacer frente a la necesidad de adaptarse a un mundo en mutación, y al mismo tiempo preservar los modos de vida que amamos los americanos, esa adaptación ha de conseguirse mediante el consentimiento de los ciudadanos. Para lograr ese consentimiento, van a ser cruciales la opinión pública y las influencias que la afectan. Creemos, en segundo lugar, que para que nuestros dirigentes puedan lograr ese consentimiento hará falta tener un conocimiento sin precedentes de la opinión pública y de los medios a su alcance para lograr ese consentimiento. Para ello, la política pública, más que nunca, habrá de tener en cuenta las necesidades y las predisposiciones del público. Creemos, en tercer lugar, que hoy en día ya tenemos, a nuestra disposición, métodos de investigación que nos pueden informar, con fiabilidad, acerca de la opinión pública y de cómo está siendo influenciada —o puede serlo en el futuro- en relación con los asuntos públicos. Vamos a elaborar estas tres ideas.

La opinión pública, hoy por hoy y en los años venideros, está sujeta a tensiones peculiares. La rapidez y la complejidad de la marcha de los acontecimientos apabullan la mente, amenazan el juicio ecuánime y tienden a hacernos cuestionar valores, suposiciones y acciones convencionales confirmadas por los hábitos en otros tiempos menos expuestos, violentamente, al cambio. Los instrumentos 
modernos de la comunicación — prensa, radio y cine- han introducido nuevas complicaciones y nuevas potencialidades en nuestra sociedad, cuya importancia entendemos sólo de modo tenue. Nuestra finalidad es la de esclarecer las maneras y los modos mediante los cuales — dada la necesidad del cambio — la opinión pública puede, del modo más efectivo, ser ayudada a adaptarse a tiempo al cambio necesario - a volver a evaluar las creencias prevalecientes, a descartar aquello que ha llegado a ser irrelevante y obsoleto, a retener aquello que sigue siendo fundamental- . Para esta finalidad, es importante que nuestros dirigentes sepan más acerca de la naturaleza de nuestras creencias, de sus elementos estables e inestables y de las influencias que las afectan. Las actitudes referentes a la política pública, las reacciones actuales y potenciales a influencias variables, han de ser filtradas y evaluadas ya no mediante métodos improvisados, sino sobre una base científica avalada por hechos comprobados de manera acertada.

Nuestra segunda creencia tiene que ver con la relevancia de la investigación respecto de la política pública. Un gobierno que se fundamenta en el consentimiento también se apoya en el conocimiento acerca de cómo conseguir, de la mejor manera posible, aquel consentimiento. Las políticas que no admiten fundamentos verdaderos para su cuestionamiento corren el riesgo de ser derrotadas, a no ser que se tenga en cuenta la predisposición y la necesidad del público, a no ser que la interpretación de los propósitos y del resultado probable de esas políticas sea comunicada de modo acertado y efectivo. La investigación en el campo de la comunicación de masas es una nueva arma segura para alcanzar este fin.

Pero ninguna investigación, por científica que sea, será relevante si no se enmarca dentro de un contexto. Con el fin de otorgar, a lo que sigue, un marco de referencia que no sea sólo vagamente teórico, ahora ofrecemos ciertas definiciones acerca de objetivos referidos a la política pública y de la naturaleza de los cambios que suponemos que van a significar la necesidad de una adaptación por parte del público.

En primer lugar, entonces, veamos el telón de fondo de la política pública. Como nación, estamos comprometidos con la libertad — libertad de prensa, de expresión, de reunión- La política pública estadounidense otorga el máximo nivel de control privado respecto de los canales de comunicación. Se da por supuesto que no es posible una libertad absoluta. Existe siempre un equilibrio flexible entre las esferas de lo privado y de lo público. Suponemos, entonces, que por «libertad» se entiende lo que se acaba de definir. Suponemos, a renglón seguido, que una nación fuerte es la que tiene individuos respetuosos consigo mismos y capaces de cooperar en actividades necesarias. Pero tal cooperación no debe encaminarse hacia los fines de un interés egoísta, sino a los del pueblo en su conjunto. Consideramos de suma importancia el sentimiento de "pertenencia con orgullo», un sentimiento de participación libre, responsable y constructiva. Conforme el mundo se hace más interrelacionado, los cambios se producen con una velocidad cada vez mayor. Las decisiones que hay que tomar con un máximo de velocidad afectan a sectores de la población cada vez más amplios. Que se tomen 
estas decisiones es necesario, pero también es peligroso. El individuo, cuyo bienestar es el objetivo de la democracia, tiende a ser olvidado, excepto como alguien a quien hay que alimentar razonablemente y manipular estadísticamente. Los hombres no son herramientas, ni pueden ser utilizados como tales durante mucho tiempo. La política pública, entonces, ha de determinar fines y elaborar medios hacia esos fines que ofrezcan márgenes para las aspiraciones responsables del respeto que los individuos han de sentir respecto a su propia persona.

Nos enfrentamos a una crisis doble, a una crisis inmediata de temor y a una crisis más remota de esperanza constructiva. En el momento presente, la política pública se preocupa por la movilización de los recursos nacionales y de su fuerza laboral ante peligros inminentes. La unidad nacional implica movilizar la opinión pública en la misma medida que implica la movilización de servicios y recursos. La presión de las circunstancias, por sí sola, decidirá hasta qué punto la forja de la opinión puede dejarse a los medios de comunicación de masas en manos de la empresa privada, o hasta qué punto deberá ser necesario el control gubernamental. La medida de la libertad que esperamos preservar en esta crisis será la disponibilidad de los responsables de la comunicación de masas para afianzar, entre el público, una preocupación incrementada respecto de los asuntos relevantes de la política pública. En tiempos como el actual, el gobierno deberá obtener la cooperación de la empresa privada en el campo de la comunicación, o imponer sus propios controles con el fin de asegurar los fines del propio gobierno.

Respecto de este aspecto de la política pública, nuestra única preocupación es cómo puede afectar el telón de fondo que hemos esbozado antes. Pero al margen de si la comunicación sigue siendo libre o viene a sujetarse a una supervisión y a unas restricciones incrementadas, sigue válido el principio de que el conocimiento derivado de la investigación va a ser necesario, más que nunca, a la hora de articular decisiones y descubrir maneras de lograr el consentimiento respecto de la política pública. En cualquier caso, la investigación será de suma importancia a la hora de medir los efectos que tienen, o pueden llegar a tener, las comunicaciones de masas.

La crisis más remota tendrá que ver, en parte, con las consecuencias de las decisiones y acciones actuales y, en parte, con las circunstancias mundiales que van a obligar a decisiones y acciones futuras. La movilización, eventualmente, cederá ante la desmovilización y ante todos los problemas de ajuste que, sin duda, presentará un mundo descoyuntado. Al margen del resultado final de la actual crisis en Europa, una cosa es cierta: los Estados Unidos van a tener que enfrentarse a nuevos problemas de índole moral, social y económica. Muchos de estos problemas pueden anticiparse, y se pueden formular, ya ahora, medidas para afrontarlos. Como en el caso de la actual crisis, podemos anticipar, en el futuro, unas responsabilidades muy incrementadas para el gobierno, así como la necesidad continuada de ir educando al público acerca de los asuntos de la política pública, si es que nuestros dirigentes han de lograr el consentimiento. Ante el telón de fondo de las creencias que hemos esbozado, podemos, 
ya ahora, prever la medida y la naturaleza de los ajustes de perspectiva, circunstancia y acción que van a ser necesarios. Los enormes recursos existentes de la comunicación de masas pueden influenciar profundamente la velocidad y el éxito de la adaptación en el modo de pensar de la gente. Pero, como ocurre en el caso de la crisis actual, va a ser necesaria la investigación para poder descubrir las necesidades públicas y para evaluar la respuesta del público. En la carrera contra el tiempo que ya se ha iniciado, no podemos permitirnos el lujo de menospreciar los medios a nuestro alcance para conseguir datos que podrían llegar a ser cruciales para resolver nuestros problemas.

En tercer lugar, creemos que los medios para conseguir tales datos ya existen. Las técnicas para el estudio de la comunicación llevan muchos años de existencia y se han aplicado en los campos de los estudios de mercado, la comercialización, la propaganda, la publicidad y las relaciones públicas. Los estudios que utilizan estas técnicas proporcionan datos de gran importancia para la política privada. Las técnicas, en sí mismas, son transferibles y habrían de usarse para apoyar la política pública.

La mentes preclaras del mundo de la empresa ya saben que el sentido común, así como las intuiciones derivadas de la experiencia práctica, cuando se escudriñan de cerca, se muestran equivocados en no pocas ocasiones. Los editores de una revista de ámbito nacional pensaban que el material que publicaban ejercía una atracción sobre unos lectores más susceptibles a la publicidad que los contenidos de su principal competidor. Una investigación reciente, sin embargo, mostró que estaban funcionando sobre la base de datos obsoletos, de hace quince años, y que de hecho los contenidos de las dos publicaciones ejercían la misma atracción. Convencidos de la eficacia de la política, los editores están ahora buscando medios para implementarla de modo más realista sobre la base de esta nueva evaluación atinada.

La investigación pionera ha mostrado cómo estudiar los actuales canales de comunicación — cine, periódicos, revistas, libros, panfletos, radio, discursosSabemos, por ejemplo, cómo averiguar si el flujo de la comunicación pública ha mostrado autoridad en una perspectiva equilibrada o desequilibrada, y con qué efecto. Unos estudios recientes sobre la producción cinematográfica han mostrado que los jueces, los policías y los directores de instituciones penitenciarias se proyectaban sistemáticamente de modo desfavorable. Pero estos estudios no llegaron a averiguar si esta proyección estaba teniendo algún efecto sobre las actitudes cívicas de los que acudían a esas películas. La investigación que mostró cómo las agencias de autoridad pública eran proyectadas en el cine tenía que haberse complementado con otros estudios — para los cuales existen técnicas - para descubrir cómo, de hecho, las actitudes quedaban afectadas por esta proyección. En la ausencia de tal estudio, sólo nos queda adivinar si la respuesta obraba o no en favor del interés público.

Incluso en cuanto a la política pública, el reconocimiento del valor de la investigación no es nada nuevo. En 1918, el gobierno americano se interesó por el estado de ánimo de los militares y de la población civil en Alemania. Se pen- 
saba que se podría acortar la guerra si pudiera desintegrarse el estado de ánimo del pueblo alemán. Llegaron una gran cantidad de informes al Departamento de Guerra. Circulaban rumores de insatisfacción en Baviera, en la región del Rin y en los distritos obreros de Berlín. El enorme volumen de pruebas aportadas al Secretario de Guerra era confuso hasta que se adoptó un método disciplinado de presentación. Todas las fuentes de información fiable se pusieron en común, se sopesaron y se utilizaron para la elaboración de gráficos que representaban el estado de ánimo de los alemanes. Por medio de su uso, fue posible comparar información de distintas fuentes y así orientar los esfuerzos militares y propagandísticos hacia los puntos débiles de la nación alemana. Estos gráficos, sin embargo, se elaboraron de modo poco hábil y así su valor fue mucho menor de lo esperado. Debido a las prisas y a la improvisación, incluso el conocimiento del que se disponía no pudo ser aprovechado al máximo.

Hoy en día, unos gráficos parecidos son considerados como indispensables en otros campos. Los juicios acerca de la política de inversiones, privadas y públicas, se hacen a la luz de gráficos comprensivos sobre cargamentos, producciones de hierro, transferencias bancarias y datos parecidos. Las encuestas de opinión del Instituto Americano de Opinión Pública y de la revista Fortune son otras instancias pioneras.

Como se ha dicho, hace mucho tiempo que se utilizan técnicas para descubrir datos que son vitales para la política y el beneficio privados, que son transferibles a la esfera de la política pública. Desde luego, del mismo modo que hay límites a la experiencia práctica, en el estado actual de la investigación técnica hay límites acerca de qué puede descubrirse mediante la investigación. Ciertos problemas son demasiado elusivos como para ceder ante la investigación científica. Hay problemas para los cuales no existe ningún método adecuado, ni personal cualificado. Pero, aun con estas reservas, en el campo de la comunicación siguen existiendo muchos ámbitos de enorme importancia de cara a la política pública, en torno a los cuales se pueden utilizar con habilidad métodos disponibles. El alcance y las limitaciones de la investigación, su coste y sus complicaciones, se exponen en la siguiente sección.

Con el fin de concretar, intentaremos enunciar el papel de la investigación sobre la comunicación de masas en una situación que, aunque sea puramente hipotética, sirve para ilustrar lo que implica dicho papel.

Supongamos que los dirigentes gubernamentales y los responsables de la comunicación de masas están de acuerdo acerca de la política con respecto a los grupos extranjeros en nuestro país. El público, piensan, deberá estar advertido de los peligros de las actividades subversivas por parte de los extranjeros, pero a la vez se deberá minimizar la antipatía popular contra los extranjeros en general, y, sobre todo, se deberán evitar brotes de sentimiento xenófobo. Entonces la 
política a la que deberán adherirse los canales de comunicación será una política que fomente la conciencia pública del peligro de las acciones subversivas, pero que a la vez fomente la tolerancia respecto de los extranjeros en general.

Supongamos que algún programa radiofónico dominical vespertino, conocido por la atracción que ejerce sobre un número considerable de ciudadanos, incluye una alocución sobre los peligros de las actividades subversivas por parte de los extranjeros. La intención explícita del locutor, de sus patrocinadores y de las emisoras que emiten la alocución es simplemente la de apoyar la política esbozada arriba, es decir, llamar la atención sobre los peligros que la nación deberá conocer. Al día siguiente o a los dos días, sin embargo, los teletipos informan desde distintas zonas del país que ha habido brotes de sentimiento xenófobo contra ciertos grupos de extranjeros. Los informes acerca de las declaraciones locales relacionadas con estos brotes aluden a la alocución radiofónica del domingo por la tarde. Como resultado, existe la fuerte sospecha que hay alguna conexión entre los brotes y aquello que se dijo en la alocución del domingo.

Supóngase, además, que los responsables de la alocución original deciden que asimismo tienen la responsabilidad de reparar el daño que causaron de modo plenamente inocente. Esta decisión cobra una nueva importancia ya que la cadena en cuestión recibe, de la Federal Communications Commission, una petición de envío del texto de la alocución original. Está claro que un esfuerzo concienzudo para reparar el daño implica aprender más acerca de la naturaleza del daño. El comentario que ocasionó en la prensa muestra claramente que sus efectos se sintieron no sólo en la radio, sino en las informaciones periodísticas sobre la desafortunada alocución, en las declaraciones locales que la mencionaron, e incluso en algunos noticieros documentales muy difundidos en los cines sobre los brotes locales que surgieron tras su emisión. Entonces, ¿a qué gente hay que dirigirse si se han de remediar los efectos no intencionados de la emisión?

¿Cuáles fueron esos efectos y qué fue, precisamente, dentro de la alocución, lo que los provocó? Claramente, la emisión, por sí sola, no era la única responsable. Hubo algo en lo que se dijo que, evidentemente, se combinó con las predisposiciones de la audiencia y con las circunstancias operantes — con la fuerza de los acontecimientos y probablemente con otras comunicaciones muy difundidas - que abonó el campo para lo que vino después.

Reconociendo estas cuestiones como básicas para cualquier esfuerzo concienzudo para reparar el daño, los responsables pusieron los medios para hallar las respuestas. A cada emisora que difundió la alocución se le pide que envíe inmediatamente a la sede central de la cadena toda la evidencia posible acerca de la atención que recibió en la zona de la emisora —informaciones de prensa de la alocución misma, editoriales y discursos que se refieren a ella, reportajes sobre los brotes subsiguientes, tratamiento en la salas de cine mediante noticieros documentales, etc.-. Además, a cada emisora se le pide que encargue a sus empleados más cualificados que entrevisten a miembros de la audiencia para determinar, de la mejor manera posible, qué es lo que hubo en la alocución que propició los brotes no anticipados. En particular, se les pide a estos empleados 
que entrevisten a personas directamente implicadas en los brotes. Algunas de las emisoras, con el afán de hacer bien la parte que les corresponde, contratan a especialistas competentes de universidades cercanas con el fin de estudiar de modo más intenso las predisposiciones de los individuos que más reaccionaron a la alocución, y de intentar descubrir qué otras circunstancias, combinadas con la alocución, hicieron que actuaran de tal modo. La organización de encuestas de una agencia nacional de opinión pública también es invitada a analizar, de modo análogo, una muestra aleatoria de la población del país que incluya a gentes de la audiencia que fueron afectadas y no afectadas por la alocución, así como a gentes a quienes la alocución no les llegó, ni directa o indirectamente.

Conforme van llegando informes de todas estas investigaciones, va tomando forma un nuevo cuadro de la situación. Ante la sorpresa del locutor, sus patrocinadores y la cadena, lo que parecieron ser algunas referencias inocentes a un puñado de extranjeros que se suponía activos en actividades subversivas, fue interpretado por la audiencia como aplicable a todos los extranjeros en general y a los descendientes de países que se presumía eran hostiles respecto de los intereses y las tradiciones del país. Algunas de las entrevistas individuales y los resultados de la muestra aleatoria revelan una actitud igualmente sorprendente de hostilidad general hacia esos grupos. Algunos aluden específicamente a lo que se consideran como rasgos raciales de los grupos involucrados, otros a individuos concretos del grupo que compite comercialmente con los informantes. Otros mencionan informes aparentemente auténticos de actividades de quinta columna en países conquistados de Europa; y un número considerable de individuos se refieren a supuestas actividades de esta naturaleza en los Estados Unidos y en América del Sur. Claramente, estaba el campo abonado en la tarde de la emisión respecto a lo que finalmente ocurrió, debido a la predisposición psicológica general de la audiencia y a la fuerza de los acontecimiento recientes.

Todo esto, claro está, contribuyó sin duda a la atención inusitada que recibió este programa radiofónico — por parte de la audiencia, por medio de los reportajes de prensa que lo comentaron, en los noticieros documentales y a través de las declaraciones locales que suscitó-. También, curiosamente, el programa en el que se insertó la alocución en este domingo concreto tenía una audiencia mayor de lo normal. Su cuota, tal como informaron las agencias de investigación de audiencias, había subido sustancialmente con respecto a su nivel acostumbrado. Ciertamente, la inclusión de esta alocución particular se había anunciado con antelación, y por coincidencia, en este preciso domingo, otro programa popular difundido normalmente a la misma hora se había despedido para el verano. Se suponía que, por regla general, este otro programa atraía más a oyentes menos instruidos, con el resultado aparente de que la audiencia que escuchó la alocución incluía, quizás por vez primera, a más oyentes de menor nivel cultural: una suposición que se confirmó mediante la desagregación de datos respecto de la audiencia en términos de status socioeconómico y al verificar la cuota de audiencia de otros programas difundidos a la 
misma hora, ninguno de los cuales superó su cuota habitual y algunos de los cuales experimentaron un marcado declive.

Por consiguiente, cómo reparar el daño se convierte en algo más problemático de lo que se pensó en un principio. Obviamente, otra alocución para contrarrestar la que había ocasionado el daño no sería suficiente. Anunciar una segunda alocución en términos comparables podría, desde luego, volver a atraer a la misma audiencia de la semana anterior. Pero, se recalca, no hay razones para suponer que la predisposición de los oyentes o la fuerza de las circunstancias fuesen, una vez más, a distraerles de sus hábitos normales como radioyentes.

Además, más o menos por las mismas razones, no hay seguridad de que una alocución contrastada fuese a acaparar la misma atención en la prensa o en las declaraciones locales. Finalmente, a estas alturas el locutor original está firmemente identificado, sin duda alguna, en la mente de los oyentes, con las opiniones que le son atribuidas acerca de los grupos extranjeros y de los ciudadanos con origen foráneo a raíz de su alocución anterior. ¿Serviría otra alocución leída por él para cambiar esa identificación? ¿Qué hacer, pues?

Ante este problema viene la sugerencia de que hay que buscar consejos de otra fuente ajena al embrollo. Si se quiere evitar que esta alocución pase a la historia como el principio de una ola de sentimientos destructivos contra todos los extranjeros y los ciudadanos de origen foráneo y así ir completamente en contra de los intereses que la propiciaron, entonces hay que planear con sumo cuidado cualquier medida rectificadora. ¿Quién hay por allí que pueda contribuir a un mejor entendimiento de lo que ha ocurrido, y quién puede sugerir qué se puede hacer a modo de remedio?

Supongamos, llegados a este punto, que se busca ayuda de un científico social que es conocido por haber estudiado los sentimientos xenófobos hacia las minorías. Cuando se le llama, parece que lleva algún tiempo anotando y analizando todo aquello que aparece en la prensa, la radio, el cine o en declaraciones públicas que parecen tener algo que ver con este tema. Indica que éste no es un episodio aislado, sino uno más de un proceso que él viene analizando desde hace algún tiempo. Justamente este tipo de sentimiento en contra de las minorías se iba gestando en el país desde años atrás. El aumento del sentimiento antisemítico en este país ya era plenamente reconocido, y ahora parecía que los mismos sentimientos se iban trasladando hacia otros chivos expiatorios.

Según su análisis, el desarrollo reciente de la comunicación de masas había reflejado esta tendencia general. La expresión "quinta columna», utilizada ambiguamente en la Guerra Civil española, estaba muy extendida. De hecho, los Aliados no habían perdido ocasión para acentuar este concepto, como, por ejemplo, con el uso del nombre del Comandante Quisling. Al mismo tiempo, iban llegando informes de actividades de quinta columna en los países conquistados, apoyados en tiempos aún más recientes por revelaciones de actividades semejantes en América del Sur. Por tanto, el sentimiento pro-Aliados en este país, apoyado por una mayor predisposición de temer y de sentir animosi- 
dad respecto de los grupos minoritarios, llevó a una excesiva generalización de los comentarios vertidos en la alocución.

Tales sentimientos — podría seguir diciendo el especialista- encontrarían más eco entre los grupos menos instruidos y menos conscientes de su propia identidad. Además, estos grupos — en su opinión - tendrían menos visos de responder ante cualquier reclamo intelectual diseñado con la finalidad de contrarrestar los efectos de la alocución anterior. Deberá tenerse esto en cuenta — advierte - en la planificación de cualquier medida reparadora. De hecho, duda de la eficacia de cualquier esfuerzo por remediar la situación. Más bien, ya que la alocución en cuestión no era un ejemplo aislado, los esfuerzos reparadores deberán tener en cuenta todos los demás factores en los medios de comunicación actuales que tienden — tal como lo ve él- a suscitar, justamente, tales sentimientos contrarios a las minorías. Para reparar el daño - apunta - es necesario determinar a quién se va a dirigir, no sólo en términos de cobertura geográfica, sino también en términos psicológicos. Su último consejo, en este punto, es que se busque a otro especialista que haya estudiado la composición geográfica y psicológica de la audiencia a la que llegan varios tipos de comunicación de masas.

Este segundo especialista, cuando entra en escena, confirma sin titubeos que, según sus propias observaciones, el programa en cuestión alcanzó una audiencia psicológicamente diferente a la que atraía normalmente. Alcanzar esa audiencia, en su opinión, requiere prestar mucha atención a los hábitos radiofónicos, de lectura y de asistencia al cine por parte de la población afectada por el programa original. Conoce las características generales de cada segmento de esa audiencia y los tipos de emisiones, libros y películas que les atraen. Sus estudios, además, le otorgan una base para poder predecir cómo va a reaccionar un grupo cualquiera ante cualquier tipo de programa, aunque sería preciso verificar la predicción mediante un análisis cuidadoso de los efectos que se produjeron a raíz de la alocución en cuestión. También sabe que el mismo mensaje, difundido por medios diferentes, con el fin de alcanzar la audiencia apetecida, tendrá que acentuar distintos aspectos del tema que sean especialmente apropiados para el medio en cuestión. Si las medidas planeadas para contrarrestar han de ser, de verdad, un remedio, entonces sugiere con énfasis que se hagan algunas pruebas previas de las reacciones que de hecho provocan. Por lo tanto, propone que previamente se prueben las medidas reparadoras con una muestra relativamente pequeña pero representativa de la población, y que se utilice el estudio de sus reacciones como base para posibles modificaciones, antes de intentar dirigirse a un público más amplio.

Así, con la ayuda de estos y de otros especialistas, empieza la tarea. Aunque se está de acuerdo con que hay que evitar la posibilidad de que se produzcan efectos no intencionados, se siguen los consejos de estos especialistas. No es cuestión aquí de sugerir la naturaleza de lo que se hace, sino sólo indicar cómo la investigación en la comunicación de masas podría contribuir al resultado. Con la ayuda de especialistas en tales investigaciones, se vuelve a determinar la audiencia originalmente afectada. Se elaboran tipos de programas de radio, 
comunicados de prensa y tratamientos en noticieros documentales, calculados sobre la base de la mejor evidencia disponible, para volver a tratar el tema de un modo adecuado, con la finalidad de contrarrestar los efectos de la alocución original. Sin duda, se prepararía una explicación que leería el locutor original, pero se contaría también con otros locutores que gozan de una posición y una identificación con la opinión pública, por lo que su papel tendría una influencia mayor. Se prueban de antemano todos los materiales preparados, tal como se sugirió y con poco coste - de hecho proporcionalmente mucho menor que el de comprobación del mercado para nuevos productos-. El esfuerzo concienzudo les ha llevado tan lejos, que los responsables están de acuerdo en la necesidad de más pruebas para comprobar los efectos reales de lo que han ideado a modo de remedio. Por consiguiente, se hacen planes antes de la campaña con el fin de hacer un seguimiento de su marcha.

Un final feliz de esta fábula podría, probablemente, adoptar la forma de una serie de gráficos que aliviaran la conciencia de todos los involucrados, al mostrar - conforme avanza la campaña- un descenso consistente en todos los índices de hostilidad abierta contra los grupos hacia los cuales se habían dirigido los brotes de sentimiento xenófobo.

Si esta fábula ha de tener además una moraleja, puede que ésta venga cuando todos los involucrados en la toma de conciencia, que surge a raíz de su éxito, piensen un poco acerca de su experiencia. El locutor original, los patrocinadores y los radiodifusores siguen convencidos de su inocencia inicial. Pero están un tanto atormentados por ciertos recuerdos. Uno de ellos recuerda, por ejemplo, que sugirió que se hiciera más propaganda en favor del programa original sobre la base de que era especialmente oportuna la alocución que se estaba preparando. Otro recuerda que el tema de la alocución surgió a raíz de una sugerencia de un conocido que ocupa un puesto relevante en una organización que — supuestamente por razones patrióticas - llevaba algún tiempo apoyando la idea de ejercer mayores controles sobre los extranjeros asentados en el país. Al final, el sentimiento de todos es que, por muy inocentes que fuesen sus intenciones conscientes, también ellos, como sus conciudadanos, compartirían las mismas predisposiciones en la planificación de la emisión, y respondieron ante la fuerza de las circunstancias de la misma manera que los oyentes. Está bien, entonces, que se contraste la intención consciente mediante medidas más objetivas, cuando los instrumentos usados son tan poderosos en cuanto a su influencia, como es el caso de las comunicaciones modernas. De alguna manera, el mero hecho de haber introducido medidas más objetivas en el juego parece haber agudizado su sentido común y haberles hecho ser más cautos respecto del futuro. Si una investigación similar llevada a cabo al principio les hubiese iluminado anteriormente, ¿habría ocurrido todo esto? ¿Habría hecho falta correr el riesgo de que las inevitables demoras en la reparación del daño causado hicieran que sea imposible repararlo completamente? Tal vez, concluyen, en medios como la radio en los que la «réplica instantánea» es a menudo difícil, hay que tomarse más molestias para evitar equivocaciones como ésta. 
Esta fábula, hay que reconocerlo, puede parecer que exagera la importancia de la investigación en el campo de las comunicaciones de masas. Ciertamente, por regla general, el sentido común, el elevado nivel de excelencia de las empresas de comunicación y los controles de la regulación legal y administrativa son suficientes para asegurar que el uso de los medios de comunicación vaya en pro del interés público. Por regla general, es posible que la aplicación de este uso de modo razonable sea suficiente como para que se lleven a cabo acciones mediante ensayos y reglas más o menos comprobadas. Sin duda, una situación crítica, como la que hemos imaginado, arroja luz sobre consideraciones de alto relieve que, si bien siempre están presentes, casi nunca parecen tan urgentes. Pero la crisis, como implica la derivación de la misma palabra, obliga a hacer un juicio; y una solución deseada de una crisis requiere que sea un juicio acertado. La situación crítica de nuestra fábula, entonces, más que exagerar, tal vez sólo pone en perspectiva las consecuencias del uso equivocado de los medios de comunicación, así como la ayuda que puede proporcionar la investigación para evitar ese uso equivocado.

Por supuesto, la situación esbozada podría fácilmente haber ocurrido. De hecho, algo muy parecido a ella fue el caso de la emisión de Orson Welles, «La guerra de los mundos», en octubre de 1938. El estudio que hizo de esta emisión el Proyecto de Investigación de la Radio de Princeton (Princeton Radio Research Radio Project), La invasión de Marte, representa, en gran medida, el mismo tipo de investigación que sugerimos en nuestra fábula. Puede que, entonces, la fábula sólo se aparte de la realidad en la suposición de que los especialistas fueran capaces de hablar a partir de conocimientos reales. Éste ciertamente habría sido el caso si los avances en la investigación en las comunicaciones de masas fueran más depurados de lo que son actualmente. Las técnicas para adquirir el conocimiento que se les atribuye a los especialistas en la fábula han sido comprobadas y se muestran efectivas. Lo único que queda para asegurar el conocimiento que estos especialistas desplegaron es otorgar una aplicación más amplia a esas técnicas, en comparación con lo que ha ocurrido hasta ahora.

De todos modos, la fábula habrá servido al fin apetecido si muestra claramente cuál es el papel que ha de desempeñar la investigación en el campo de las comunicaciones de masas. En una palabra, ese papel es el de aprender qué es lo que hacen las comunicaciones de masas en nuestra sociedad. Aprender lo que hacen implica conseguir evidencia que nos permita contestar a cuatro preguntas básicas, cada una de las cuales entra en juego en la situación hipotética de la fábula. Como quedó patente en ese caso, lo que hacen se convirtió en una cuestión de qué efectos producen los medios de comunicación en su conjunto, o produce un solo medio en particular. Los efectos que producen, además y de modo inevitable, implicaban descubrir a quién se dirigió el mensaje. La manera en que ocurrieron esos efectos hace necesario el análisis de qué es lo que se dijo. Y ese análisis, si ha de ser completo y propiamente ilustrativo, requiere que se conteste a una cuarta y última pregunta: quién lo dijo y cuál era su intención. En una palabra, entonces, el papel de la investigación en las 
comunicaciones de masas es determinar quién, y con qué intención, dijo qué, a quién y con qué efectos.

De hecho, por supuesto, cada una de las preguntas subsidiarias implicadas en el problema en su conjunto ha de ser contestada en contextos psicológicos y sociales. Quién lo dijo pide que vayamos mucho más allá de quién era el locutor. ¿Qué identificación conlleva en las mentes de los que le escuchan? ¿Tienen sus palabras una influencia positiva o negativa para ellos -o para quiénes entre ellos—? En una palabra, ¿qué representa psicológicamente para los que le escuchan? $\mathrm{O}$, en un contexto social, ¿para quién habla? ¿A qué propósitos sirve cuando habla? ¿Qué influencias dan forma no sólo a lo que dice, sino a la probabilidad de que lo diga? En una palabra, ¿¿de qué parte de la estructura de la sociedad es representativo el locutor, sea de forma intencionada o no intencionada?

Las respuestas a la pregunta acerca de lo que se dijo, de igual modo, han de buscarse en un contexto psicológico y social. El contexto psicológico surge del conocimiento de cómo tienden a reaccionar los individuos ante determinados estímulos. A partir de un conocimiento general, los especialistas en la materia pueden, con relativa certeza y de forma objetiva, identificar los factores que, en una comunicación cualquiera, tienen mayores visos de evocar una reacción generalizada. Así, el análisis de lo que se dijo se convierte en la tarea de tomar nota de la ocurrencia y recurrencia de tales factores - o símbolos, como los llaman algunos especialistas- Su significado en una cierta comunicación está determinado, en parte, por su ocurrencia y recurrencia en el flujo general de la comunicación de masas. En un contexto social, entonces, la finalidad es la de relacionar lo que se dice en una comunicación cualquiera con el flujo general de la comunicación.

La tarea de responder a la pregunta de a quién se dirigió el mensaje sigue una pauta parecida. El tamaño de la audiencia, así como su ubicación geográfi$\mathrm{ca}$, desde luego, son de suma importancia, pero casi igualmente importantes son las características psicológicas de la audiencia, sus predisposiciones generales y sus predisposiciones en el momento preciso, en la medida en que sea posible averiguarlas. Una vez más, cobra importancia el contexto social por medio de la influencia que ejercen las circunstancias pasadas y presentes en el individuo.

Sólo cuando se contesta, por lo menos tentativamente, a estas tres preguntas es cuando se está en condiciones de tratar la cuarta pregunta, que de alguna manera es la pregunta clave: los efectos de lo que se dijo: ¿con qué efecto psicológico, breve en el tiempo o duradero en cuanto a su influencia? ¿Cuáles fueron sus efectos en el comportamiento individual, o en las manifestaciones que muestran cambios en los estilos de vida o incluso en las instituciones de la sociedad?

Cada una de estas cuatro preguntas juega un papel en la situación hipotética esbozada más arriba. La tesis de este memorándum es que las respuestas a estas preguntas tienen una utilidad práctica en el uso de las comunicaciones de 
masas y en su control acertado de cara al interés público. La investigación ya ha recogido pruebas de las que se pueden deducir algunas respuestas a estas preguntas, aunque sean provisionales. Sabemos, por ejemplo, por la investigación industrial y a partir del trabajo de las Oficinas de Investigación de la Radio de Princeton y Columbia, algo sobre el tamaño, la ubicación geográfica y - por lo menos de modo esquemático- la composición psicológica y sociológica de la audiencia de oyentes de la radio. Pero queda aún por acumular y sistematizar un conocimiento general que sea fiable a los fines de la deducción. Con esa finalidad, lo que se necesita ahora es llevar a cabo estudios específicos que intenten contestar estas cuatro preguntas básicas en relación con comunicaciones determinadas o con series de medios. Las técnicas para lograr estas respuestas ya han sido comprobadas en este campo y en otros. Si se encuentran los medios para aplicarlas en mayor profundidad en el campo de las comunicaciones de masas, los resultados podrán establecer la calidad de la tarea. La siguiente sección, en la que se describen las técnicas ya disponibles, posiblemente sirva para esclarecer aún más lo que hay que hacer.

\section{III}

Las técnicas disponibles para la investigación en las comunicaciones de masas son, esencialmente, aquellas que se han aplicado con eficacia en otros campos a lo largo de las últimas décadas. Muchas llevan por lo menos dos decenios ejerciendo una influencia en la formulación de políticas privadas, particularmente en los estudios de mercado. Hoy en día, pocos productores ponen sus mercancías a la venta sin usar estas técnicas para explorar previamente el mercado, y la mayoría de ellos, en el momento actual, se fían completamente de los hallazgos de tales técnicas a la hora de determinar cuál va a ser su política de comercialización. La fiabilidad de estas técnicas a tales efectos se ha demostrado, repetidamente, en la forma de incrementos en los beneficios.

Además, muchas de las mismas técnicas se han utilizado en la investigación sociológica y psicológica para obtener hallazgos que ya no se cuestionan seriamente. Pero en estos campos, igual que en el campo de la comunicación de masas, la aplicación de tales técnicas, más que nada, tiene que ser controlada de un modo más estricto que en los estudios de mercado. Allí, evidentemente, las finalidades de la investigación y su contexto psicológico y social pueden enunciarse definitivamente en términos de la operación comercial y sus motivos.

El escepticismo existente en algunos casos acerca de la fiabilidad de estas técnicas cuando se aplican en un contexto psicológico o social se basa, posiblemente, en la falta de entendimiento en torno a cómo se controla su aplicación. Es probable que este escepticismo se centre en torno a la habilidad de unos investigadores concretos a la hora de aplicarlas dentro del marco de controles predeterminados. De hecho, la formación de investigadores en estos campos, cuando es adecuada, les disciplina en este tema concreto del control, al hacer- 
les conscientes de su relación entre su condición de recopiladores de datos y aquello que están intentando describir. En términos más técnicos, el recopilador de datos ha de considerarse como un observador, y aquello que intenta describir es su campo de observación.

Desde su posición como observadores, los investigadores en el campo de las comunicaciones ya conocen ahora muchas maneras de describir los elementos importantes de este campo: las actitudes de los que emiten mensajes y de los que los reciben, los contenidos de los canales de comunicación y los efectos que ocasiona el flujo de comunicación. En otras palabras, saben lograr las respuestas a las cuatro preguntas básicas que enunciamos en la sección anterior.

Las técnicas que se vayan a usar varían en cuanto a las relaciones que se establecen entre el observador y su campo de observación. Se pueden clasificar grosso modo, si embargo, conforme a dos variables: la duración de la observación en el tiempo y la complejidad del método. El observador puede centrar la atención en torno a un acontecimiento durante un período de tiempo corto o largo. Los métodos de observación empleados pueden ser simples o altamente complejos. A veces, se pueden utilizar procedimientos sencillos para una observación prolongada; con menos frecuencia, se pueden utilizar métodos complejos para una observación breve en el tiempo. Pero, a los fines de una descripción cabal, es conveniente pensar en una escala que va de la técnica breve y sencilla, que debido a que se aplica a múltiples casos podríamos denominar «extensiva», a la técnica prolongada y compleja, que debido a que su aplicación se limita a pocos casos podríamos denominar «intensiva».

Dos ejemplos conocidos de técnicas de encuesta pueden ilustrar los extremos de esta escala. El recopilador que, en una sola ocasión, pregunta a un número de personas si oyeron la alocución radiofónica del Presidente Roosevelt, está utilizando un método extensivo de observación (es decir, corto y sencillo). El psiquiatra que somete a sus pacientes - necesariamente pocos- a un estudio psicológico profundo mediante entrevistas diarias a lo largo de varios meses, está utilizando una técnica intensiva (es decir, prolongada y compleja).

Esta misma amplitud entre lo extensivo y lo intensivo existe no sólo entre los métodos para estudiar los emisores y receptores de comunicaciones y los efectos de las comunicaciones, sino también entre las técnicas que se usan para describir lo que se está comunicando. El alcance y los métodos de tales estudios pueden variar desde una clasificación rápida y superficial de las palabras usadas en unos cuantos panfletos enviados a unos electores, hasta un análisis sistemático y altamente sofisticado de los símbolos diseminados por todos los canales principales de la comunicación de masas en un área determinada durante un largo período de tiempo.

La selección de una técnica determinada para cualquier tarea en la investigación de la comunicación está determinada principalmente, claro está, por los objetivos de la investigación, la competencia y el ingenio de los observadores disponibles, así como por la cantidad de recursos financieros involucrados. Todas las técnicas desarrolladas en los amplios campos de la investigación 
social, psicológica y comercial son aplicables a la investigación de la comunicación, pero según grados variables de aplicabilidad y validez. A los fines de la concreción, no obstante, vamos a centrarnos, en este memorándum, en torno a cinco técnicas importantes que podemos ya calificar como aplicables para la investigación en el campo de la comunicación.

Cuatro de estas técnicas son aplicables principalmente al estudio de aquellos que difunden y reciben comunicaciones, y a los efectos propiciados por el flujo de comunicación: primero, la encuesta o entrevista corta; segundo, la consulta o repetición de entrevistas a los mismos encuestados; tercero, la entrevista intensiva; y cuarto, los estudios comunitarios. La quinta técnica consiste en el análisis sistemático del contenido de las comunicaciones para poder comparar las distintas comunicaciones y la medición de las fluctuaciones en su flujo total.

Estas y otras técnicas están disponibles para la investigación en la comunicación de masas: disponibles en el sentido de que no sólo se entiende su valor y sus limitaciones particulares, sino de que son viables económicamente y de que se sabe que su coste es razonable, y de que se pueden lograr los servicios de investigadores de suficiente competencia para su aplicación.

Como se ha dicho, el escepticismo acerca de la fiabilidad de las técnicas existentes parece centrarse en torno a la duda respecto de la capacidad de cualquier observador, por muy desinteresado y sincero que sea su propósito, de medir adecuadamente la opinión de otra persona, o incluso de evitar influenciar las opiniones de los demás por sus preguntas acerca de esas opiniones. El escéptico, tal vez, sólo se imagina al encuestador tocando el timbre, o con libreta en mano en la sala de estar del ama de casa. Igualmente importante, y aún más, es el mecanismo total de la técnica, de la que el encuestador es únicamente el agente.

Obviamente, los grados de habilidad, formación, disciplina y percepción necesarios para la aplicación válida de cualquier técnica varían con la complejidad y la sofisticación de la propia técnica. Los estudios que se apoyan en técnicas extensivas, desde luego, habrán de ser planeados y supervisados por observadores que gozan de muchos conocimientos — no sólo de la metodología, sino del contexto psicológico y social- y de una percepción total de su relación con su campo de observación. Bajo tal supervisión, la tarea de recopilar datos, normalmente, puede llevarse a cabo por observadores relativamente poco sofisticados, cuya fiabilidad puede asegurarse mediante controles de distinto tipo.

La técnica de la encuesta ilustra este caso. Los que diseñan y administran los estudios de encuesta deberán tener un alto grado de destreza en el uso de la técnica de la encuesta como herramienta. Deberán poseer, además, por lo menos un conocimiento general del contexto psicológico y social en el cual se va a aplicar el método de la encuesta. Esto es necesario no sólo para elaborar las preguntas, evaluar las respuestas y analizar las reacciones, sino especialmente para elaborar muestras representativas a la hora de llevar a cabo las entrevistas. Debido a que normalmente es imposible llevar a cabo un censo completo 
de todos los miembros del grupo que se está investigando, hay que fiarse de muestras que sean representativas de todo el grupo en su conjunto según aquellos criterios que se consideren importantes. La elaboración de tales muestras claramente requiere un amplio conocimiento de la estructura social.

Con un marco general tan sofisticado, unos observadores relativamente poco sofisticados pueden ser utilizados como entrevistadores. La competencia y la fiabilidad de su trabajo pueden asegurarse adecuadamente no sólo mediante la planificación, la formación y la asignación de instrucciones cuidadosas, sino mediante los rigurosos medios de seguimiento de la consistencia interna y de la verificación estadística. Hoy en día, la fiabilidad del método de la encuesta, propiamente administrada, está muy establecida en cuanto a los fines algo limitados para los que se acomoda y dentro de límites estadísticos de veracidad que se determinan con facilidad*.

Esto no quiere decir que los procedimientos de encuesta acertados pueden ser llevados a cabo por aficionados, sino que en el marco de una planificación y una supervisión al estilo de lo que ya disponemos entre el personal del American Institute of Public Opinion y otras organizaciones reconocidas que ela-

* Existen pruebas persuasivas, aunque no necesariamente conclusivas, de este hecho, que se pueden hallar en el acierto con que el American Institute of Public Opinion predijo siete resultados electorales en 1938 (los últimos pronósticos que va a publicar este Instituto hasta las inminentes elecciones de 1940):

TABLA 1

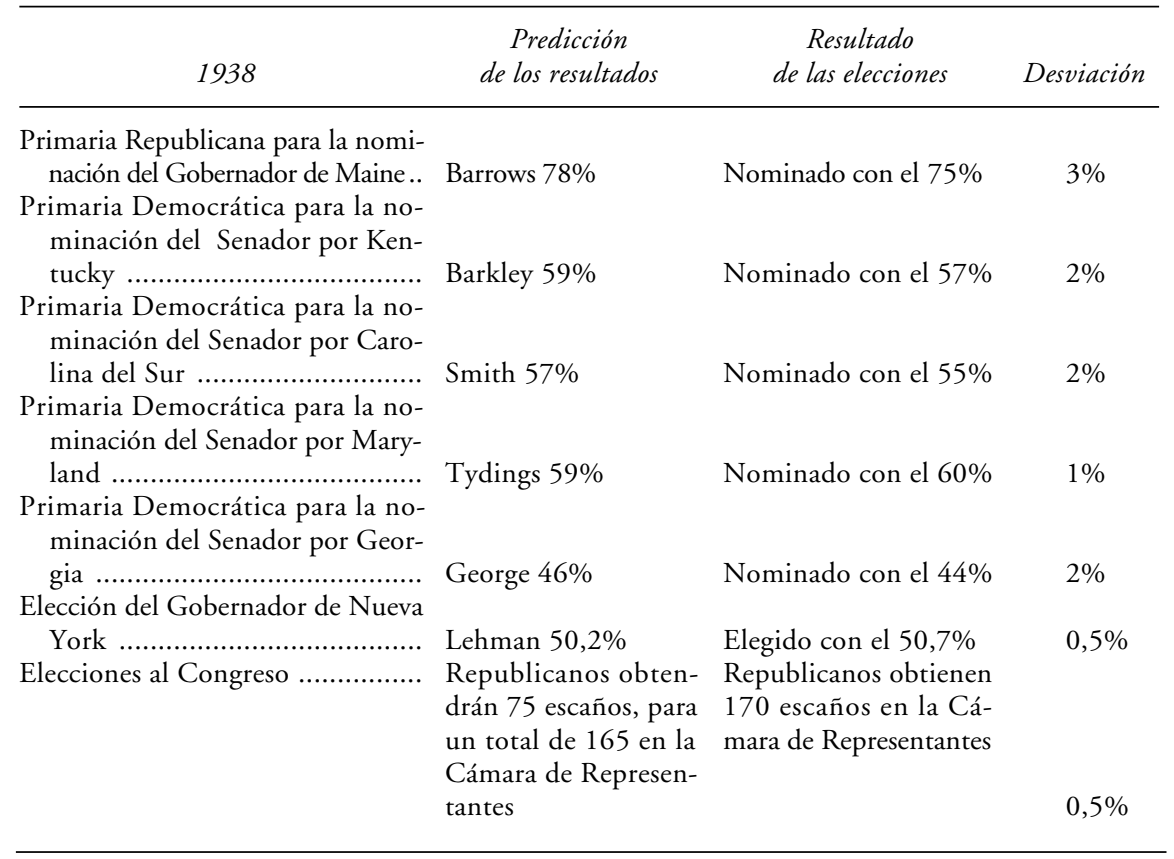


boran encuestas, uno puede fiarse de observadores semiprofesionales para la recopilación de datos extraídos de entrevistas cortas. También puede decirse que la técnica de la encuesta ha sido desarrollada, en la práctica, hasta tal punto que parece que se han resuelto los principales problemas metodológicos, y por tanto es adecuada para lograr resultados fiables del tipo relativamente poco elaborado que se obtienen con los métodos extensivos, cuando la técnica de la encuesta se aplica dentro del marco de controles predeterminados bien comprendidos.

Por otra parte, la técnica del panel como herramienta de investigación sigue en fase experimental. El método del panel es un ejemplo de una técnica a mitad de camino entre la encuesta extensiva y los procedimientos psicológicos aún más intensivos. Implica la observación continuada del mismo grupo de sujetos mediante entrevistas repetidas durante un período de tiempo. Igual que lo que es normalmente el caso con los estudios mediante encuestas, se cuida la selección del grupo de consulta para lograr una muestra representativa según los criterios que se consideran importantes a los efectos del estudio.

El método del panel es más intensivo que la técnica de la encuesta, y normalmente requiere más destreza en su administración. Los que planean y supervisan estudios de panel deberán gozar de un alto grado de sofisticación desde el punto de vista metodológico, social y psicológico. Esto es especialmente cierto cuando se utiliza el panel para aquello que se le acomoda muy bien: el estudio de los efectos de la comunicación. Porque, en este caso, las pautas más complicadas de causalidad a menudo están operando, y hay que extremar la precauciones y desplegar mucha destreza para hacer un seguimiento de las influencias causantes. Los mismos controles rigurosos, con todo, deberán aplicarse en el uso de la técnica del panel, igual que con la encuesta: planificación inteligente, instrucción cuidadosa, formación y supervisión de los observadores, consistencia interna y verificación estadística.

Las técnicas disponibles en este nivel, a mitad de camino entre lo intensivo y lo extensivo, están en fase experimental, pero pueden adivinarse sus posibilidades por un estudio que está llevando a cabo en este momento la Oficina para la Investigación de la Radio de la Universidad de Columbia. El problema establecido era estudiar la influencia de la radio en la opinión de los oyentes. Para lograrlo de modo factible, se necesitaba una situación semiexperimental en la que un número relativamente grande de personas quedaría expuesto a un gran volumen de material de peso en torno a una determinada temática. Se escogió la presente campaña presidencial como temática. Luego, el estudio tenía que ser ubicado de tal manera que se asegurara una alta probabilidad de que el material radiofónico tuviera suficiente peso. Por esta razón se eligió un condado poco definido políticamente, que en el pasado había fluctuado considerablemente en sus simpatías electorales.

Más allá de esto, se necesitaba encontrar grupos de personas que se suponía que podrían ser influidas por el material radiofónico, y que esta influencia fuese significativa en el contexto social. Así que se escogieron dos grupos para el estu- 
dio, cuyos miembros no estaban unidos por razones de afiliación política y cuyo peso numérico era lo suficientemente grande como para tener importancia dentro del conjunto de la población: los artesanos y los comerciantes.

El siguiente paso fue elaborar una muestra representativa de estos grupos, que llegaron a sumar unos 3.000 sujetos, a los cuales se les aplicó una encuesta acerca de sus preferencias presidenciales con el fin de averiguar la configuración de los dos grupos en su conjunto. Dentro de los dos grupos se escogieron, luego, a individuos para confeccionar una muestra que incluyera un considerable número de personas que no eran ni Republicanos ni Demócratas convencidos, sino que habían cambiado en cuanto a sus preferencias políticas en el pasado, de tal modo que se aumentara la probabilidad de que pudieran cambiar de opinión durante la presente campaña presidencial.

La representatividad de la muestra de la consulta se verificó después mediante la comparación de las respuestas de sus miembros con las de la población en su conjunto. En el momento presente, la muestra del panel está siendo estudiada intensivamente mediante una serie de ocho entrevistas espaciadas en el tiempo. Se está recopilando la información cumulativa acerca de los atributos sociológicos y psicológicos de los miembros del panel, así como información acerca de sus opiniones políticas en cada momento. Siempre que un miembro del panel revela que ha cambiado de opinión, se envían observadores altamente formados para entrevistarle intensivamente con el fin de descubrir los factores que han incidido en este cambio.

Se intenta evitar la posibilidad de que el mismo proceso de entrevistar a los miembros del panel, una y otra vez, vaya, en sí, a ejercer una influencia sobre sus opiniones, mediante el uso de tres grupos de control, cada uno de los cuales fue entrevistado al inicio del estudio, y que a su vez será entrevistado de nuevo a intervalos regulares durante la campaña, con el fin de determinar si el grupo del panel está manifestando desviaciones en sus opiniones que sugieren que el proceso de entrevistas repetidas está, o no, ejerciendo una influencia.

Finalmente, los resultados del panel serán contrastados con los resultados electorales regionales, y éstos, a su vez, con los resultados nacionales. No sólo servirá esto como un control para el estudio del panel, sino que servirá asimismo para situarlo en su contexto social apropiado.

Aquí, una vez más, la planificación cuidadosa, la supervisión competente, la instrucción detallada y la formación de los entrevistadores, así como los controles de consistencia interna y de verificación estadística, aumentarán la seguridad en la fiabilidad de los resultados. En cuanto a los aspectos de las técnicas de encuesta y a la hora de llevar a cabo las entrevistas rutinarias, se pudieron utilizar, con seguridad, entrevistadores menos sofisticados. Para las entrevistas que giran en torno a los factores que hacen que la gente cambie de opinión fueron necesarios entrevistadores más habilidosos; pero estaban disponibles y habían realizado cursos especiales de formación.

Así que, tanto en el caso de las técnicas de encuesta como en las de panel, el problema de la disponibilidad (aparte del coste) es, en gran medida, encon- 
trar observadores competentes y especializados para planear, gestionar y supervisar tales estudios. Por lo demás, pueden emplearse investigadores menos sofisticados, disponibles en gran cuantía, dentro de un marco de controles predeterminados.

Con estudios de gran profundidad psicológica, sin embargo, la situación es muy diferente. Supongamos, por ejemplo, que el problema es descubrir información significativa acerca de la personalidad de unos sujetos con el fin de mostrar relaciones entre sus actitudes y su personalidad. Presumiblemente, la reacción de unas personas predominantemente ansiosas respecto de la guerra va a ser diferente de la de aquellas en las que las tendencias agresivas dominan y que tienen un perfil de ansiedad bajo. En estudios de este tipo, cada observador deberá ser altamente competente, no sólo en cuanto a formación y destrezas, sino también en cuanto a su conocimiento del contexto social y psicológico. Porque en este caso poco hay para verificar la fiabilidad excepto la competencia, integridad y disciplina del propio observador. Huelga decir que, en este nivel, sólo hay un puñado de observadores realmente competentes disponibles.

La cuarta técnica, a la que nos referimos antes como estudios comunitarios, no es, en el sentido estricto de la palabra, una técnica. Los estudios comunitarios, como se les llama ahora en la investigación sociológica, intentan descubrir cómo funciona una determinada comunidad como unidad social. A lo largo de las últimas dos décadas se han llevado a cabo varios estudios de este tipo. Tal vez el más conocido es el trabajo de Lynds en Middletown. Otro estudio más intensivo es el de Yankeetown en Nueva Inglaterra, que se publicará en una serie de volúmenes preparados por el Profesor Lloyd Warner y sus colaboradores. Como resultado de estos estudios y de otros semejantes, aunque menos conocidos, existe una gran cantidad de información acerca de la estructura y el funcionamiento de bastantes comunidades estadounidenses. Pero, salvo de modo incidental, ninguno de estos estudios, hasta la fecha, ha tratado el tema de lo que hacen los medios de comunicación en estas comunidades.

Este tipo de estudios se incluye entre las técnicas disponibles para la investigación en la comunicación porque creemos que proporciona una excelente oportunidad para descubrir lo que hacen las comunicaciones en un contexto social que, de forma inusual, puede definirse bien. Esta oportunidad puede aprovecharse mediante estudios especiales suplementarios en comunidades que recientemente han sido objeto de una investigación sociológica general, o mediante la inclusión de las comunicaciones de masas como un aspecto especial dentro de los estudios comunitarios que se llevan a cabo en el momento actual.

Lo que se puede ganar queda ilustrado por los resultados de un estudio experimental actualmente en marcha, pero que - debido a las circunstancias locales - no puede ser divulgado públicamente y ni siquiera ser identificado de forma directa en el momento presente. Este estudio se está llevando a cabo en una comunidad, típicamente en una de las pequeñas ciudades del medio 
oeste, cuyo funcionamiento como unidad social ya había sido investigado. Con el conocimiento logrado en aquella investigación previa fue posible, con relativa facilidad, averiguar cómo se configura, de modo habitual, la opinión dentro de la comunidad. Realmente, la configuración de la opinión dentro de la población de la ciudad tiene una estructura bastante definida. Cada grupo social en la ciudad incluye miembros que tienden a ser líderes de opinión. De hecho, en el caso de la opinión acerca de la guerra europea, el observador adiestrado que conduce la investigación puede demostrar, virtualmente, cómo se forma dentro de esta estructura. Conociendo, además, el flujo de la comunicación de masas al que varios miembros de la población están expuestos, por regla general, puede determinarse con relativa facilidad su influencia dentro de tal estructura.

En este estudio concreto, por ejemplo, una persona aparece como un líder de opinión clave, tanto dentro de su propio grupo social como en la ciudad en su conjunto, debido a su influencia general. Esto, combinado con el hecho de que él es, prácticamente, la única persona en la comunidad que lee ciertas revistas nacionales más serias, así como algunos periódicos metropolitanos, le otorga una importancia de cara a la investigación en las comunicaciones, que un muestreo no captaría y que un estudio individual intensivo pasaría por alto, si su posición en el contexto social no estuviera establecida.

Los estudios comunitarios, evidentemente, requieren observadores con especial competencia para la planificación, gestión y dirección. Con una guía adecuada en estos aspectos - y hay especialistas que la pueden proporcionarel trabajo puede ser llevado a cabo por observadores menos adiestrados: preferiblemente estudiantes de postgrado con buena formación antropológica, sociológica y psicológica, con experiencia previa en trabajos de campo de este estilo. Con una supervisión adecuada, un observador así, trabajando conjuntamente con personal de la comunidad, podría, en la mayoría de los casos, lograr lo que se busca.

De estas cuatro técnicas, la encuesta y el panel trabajan con muestras seleccionadas de tal modo que sean representativas según los criterios aceptados del muestreo. Pueden omitir, no obstante, a personas que tipifican consideraciones de importancia para la investigación en la comunicación, particularmente consideraciones que se refieren a sus contextos psicológicos y sociales. Para tales consideraciones, las técnicas tercera y cuarta — los estudios psicológicos intensivos y la investigación mediante estudios comunitarios - cobran una especial urgencia. Porque es de estos estudios de donde vamos a sacar tales consideraciones; por ejemplo, que las personas ansiosas en el sentido psicológico tienden a reaccionar ante las comunicaciones de una cierta manera, o que la opinión en una comunidad tiende a ser canalizada por medio de algunos principales líderes de opinión. Por lo tanto, las técnicas tercera y cuarta, al tratar de modo más o menos intensivo a encuestados individuales y en torno a situaciones sociales concretas, proporcionan fuentes valiosas para hipótesis que vale la pena verificar mediante los procedimientos más estadísticos de los estudios de 
encuesta y de panel, y para lograr datos más refinados que ayuden a explicar los resultados estadísticos.

Por regla general, estas cuatro primeras técnicas, como se ha dicho, tienen en cuenta lo que se comunica sólo en tanto que produce algún efecto. La quinta técnica, a la que nos referimos antes como el análisis sistemático del contenido de la comunicación de masas, tiene la finalidad de colocar lo que se comunica dentro del flujo general de la comunicación, diseminada tanto a través del mismo medio como a través de otros medios. Llama la atención que se haya hecho tan poco para analizar este flujo, y que tan pocos se preocupen por el modo en que las personas afectadas por las comunicaciones de masas manifiestan una preocupación correspondiente acerca de los elementos en las comunicaciones ante los que, supuestamente, está reaccionando la gente.

Esencialmente, analizar los contenidos de la comunicación de masas es una tarea de clasificación de tales elementos de modo sistemático. El grado de refinamiento con el que habrán de ser definidos depende, desde luego, de las finalidades del estudio en particular. Ejemplos conocidos de clasificación sencilla de materiales impresos - no para los fines de la investigación en la comunicación, sino para la comodidad de la referencia- son los distintos sistemas (como el Sistema Decimal de Dewey) que se usan en las bibliotecas para describir, según temas, los contenidos de las publicaciones conforme a algún esquema predeterminado. De hecho, su uso en los catálogos de las bibliotecas proporciona un tipo de índice comprensivo de contenidos en cuanto a los materiales impresos. De estos catálogos, o de los varios índices periódicos que usan una clasificación similar, puede seguirse el flujo de material impreso acerca de cualquier tema a lo largo del tiempo. Pero tal seguimiento, a no ser que se consulten los trabajos a los que se refiere, no será más discriminatorio que las sencillas clasificaciones que se realizan en la catalogación. Por regla general, a los efectos de la investigación sobre la comunicación, habrá que utilizar sistemas de clasificación más detallados y más refinados para describir aquello ante lo cual reacciona la gente.

Con pocas excepciones, el único análisis actual de los contenidos de la comunicación se da en el trabajo de las agencias de recortes de prensa, algunas de las cuales incluyen, en su cobertura, aquello que se dijo en la radio y en los noticieros documentales en las salas cinematográficas. Sin embargo, en su práctica, la clasificación es, cuando menos, tosca: unos empleados no cualificados anotan simplemente, de modo mecánico, la ocurrencia de cualquier nombre o tema específicamente señalados, muy al estilo de la concordancia, que toma nota de la ocurrencia de una palabra. Si el flujo de la comunicación ha de ser sujeto a un seguimiento a partir de su trabajo, ese seguimiento, por necesidad, quedaría limitado por este procedimiento mecánico y tosco.

Ha habido, con todo, algunas instancias aisladas de un estudio más incisivo que establece, con cierta garantía, su viabilidad. Lasswell, por ejemplo, en su "Técnicas propagandísticas de la Guerra Mundial», elaboró una clasificación amplia que mostró tendencias significativas, en su aplicación a la propaganda 
de aquella guerra. El valor de tales estudios puede ilustrarse con el trabajo de uno de los estudiantes de Lasswell, que intentó un análisis similar en cuanto a los contenidos de dos periódicos estadounidense importantes durante el período que va desde el comienzo de la guerra en 1914 hasta la entrada de nuestro país en ella en 1917. Mediante este análisis, se pudo comprobar un cambio, a lo largo de ese período, de lo que el autor llamó símbolos de "ellos» en referencia a la guerra, y símbolos de "nosotros» conforme se acercaba el momento de la entrada de los Estados Unidos en la guerra. A lo largo de aquellos años se produjo una transición sutil, de la que se percataron pocas personas, desde la discusión de los acontecimientos «allende el mar», hacia la discusión en torno a lo que habría de ser «nuestra» política, conforme los Estados Unidos se acercaban, de modo gradual, desde la posición de espectador hacia la de participante activo. Claramente, ésta era una tendencia que tenía que haber notado cualquier estudioso de los efectos de la comunicación en aquellas fechas. Sin duda, hay tendencias similares en las que hay que colocar cualquier comunicación si se pretende entenderla cabalmente.

La relevancia de la clasificación adoptada para tal análisis depende de la finalidad que se pretende alcanzar con él. Los especialistas que conocen las necesidades de la investigación pueden elaborar clasificaciones satisfactorias, y el trabajo mismo de análisis puede realizarse, bajo su supervisión, por empleados menos adiestrados e incluso por personal administrativo. La elección del empleado dependerá del ingenio que se requiere en la aplicación de la clasificación adoptada.

No se obtendrá jamás un cuadro completo de lo que hace la comunicación en nuestra sociedad hasta que no se analice sistemáticamente su contenido y se anoten sus tendencias. Ya en la actualidad, el trabajo de varias encuestas nacionales está proporcionando un registro cada vez más satisfactorio de lo que son, en términos generales, los efectos actuales de la comunicación de masas. Pero hasta la fecha nadie está describiendo, con el mismo esmero, las comunicaciones que supuestamente están produciendo esos efectos. Ciertamente, nuestra comprensión de la comunicación de masas será deficiente hasta que nos acostumbremos a pensar en cualquier comunicación en su relación con la totalidad del flujo, como ya estamos acostumbrados a pensar en los individuos en su relación con toda la población en su conjunto.

Si bien cada una de estas cinco técnicas, en cierto sentido, representa una fase en la investigación en la comunicación de masas, sus plenas potencialidades sólo se alcanzarán en una investigación que esté tan organizada como para permitir que todas se centren en torno a un mismo problema. Hasta que su aplicación no esté integrada de esta manera, realmente no sabremos qué es lo que hace la comunicación. Supongamos, por ejemplo, que su aplicación está integrada en una comunidad cuyo modo de funcionar como unidad social es bien conocido. El flujo de comunicación hacia esa comunidad en cuanto a un tema determinado se analiza cuidadosamente conforme a una clasificación basada en el mejor entendimiento de ese tema que esté a nuestra disposición. 
La audiencia para cada comunicación dentro de la comunidad queda determinada, se evalúan sus efectos directos en términos psicológicos y sociales, y se anota la influencia relativa, respecto de la comunidad, de los responsables de cada comunicación. Supongamos, además, que todos estos estudios se continúen a lo largo del tiempo y que se haga un seguimiento de las fluctuaciones que se producen en ese período. Todo esto, conjuntado, proporcionaría la base para el control inteligente de la comunicación de masas en pro del interés público, cosa que no existe en el momento presente.

Tal vez el ideal actual de la investigación de la comunicación sea que se realicen, simultáneamente, varios estudios de esta naturaleza en varias comunidades a lo largo y ancho del país, que se consideren como típicas de la nación de diversas maneras. En el momento presente, el número de estudios necesarios para proporcionar una muestra adecuada para un seguimiento nacional sólo se puede adivinar; estimamos que serían necesarios unos cien estudios, aunque probablemente unos cincuenta o posiblemente unos veinticinco serían suficientes.

El coste de la investigación en la comunicación de masas no parece desproporcionado en vista del valor de los resultados. Sobre la base de la economía, lo ideal —una vez más - sería un programa de investigación en el que las distintas técnicas disponibles se conjunten de modo integrado. Una vez que se inicie una consulta, por ejemplo, la aplicación de otras técnicas diversas, dentro del mismo contexto, implican menos gasto, y sin embargo producen resultados que se pueden correlacionar fácilmente.

Aunque el coste de algunos estudios es algo difícil de evaluar de antemano, es posible hacer unas estimaciones bastante fiables del coste de la aplicación de técnicas específicas. En el caso de los mecanismos de la encuesta, por ejemplo, el tamaño de la muestra que se requiere para lograr unos resultados fiables, dentro de un margen de error de un cuatro por ciento, varía, normalmente, entre 1.675 personas en el caso de una población de 5.000 personas, a 3.000 personas en el caso de la totalidad de la población nacional. El coste de la obtención de los resultados de encuestas nacionales mediante organizaciones especializadas varía entre 100 y 150 dólares por pregunta. Por lo tanto, una encuesta que contiene entre diez y quince preguntas, aplicada a una muestra nacional de 3.000 casos, costaría alrededor de 1.000 a 1.500 dólares, si existieran mecanismos para analizar los resultados. Tal análisis, por supuesto, es un asunto puramente mecánico mediante el uso de las máquinas modernas de tabulación. Los resultados, perforados en tarjetas, se tabulan con un esfuerzo y retraso mínimos, simplemente por medio de la introducción de las tarjetas en las máquinas; y las retabulaciones y las correlaciones pueden verificar, rápidamente, cualquier hipótesis que sugieran los primeros resultados. Si se deja el análisis en manos de la organización encuestadora, el coste de una encuesta nacional, por regla general, sólo supone aproximadamente 3.000 dólares.

Sobre una base geográfica más limitada, el coste de cada entrevista con diez a quince preguntas sencillas viene a ser de 30 a 50 centavos de dólar.

El coste de cada entrevista para paneles es algo más elevado, ya que el 
entrevistador tiene la obligación de volver a localizar a las personas que ya habían sido entrevistadas. La muestra, sin embargo, es normalmente mucho más pequeña que la muestra de la encuesta — por regla general, basta con 200 a 400 casos- Los costes para las entrevistas varían de 70 centavos en ciudades pequeñas a un dólar en ciudades grandes, y tal vez hasta 1,70 dólares en zonas rurales que implican la necesidad de desplazamientos. Una entrevista inicial exploratoria cuesta entre dos y tres dólares. Entrevistas más intensivas con miembros de un panel, que requieren más destrezas por parte del entrevistador, pueden llegar a costar diez dólares cada una.

Una de las finalidades particulares de este tipo de investigación será siempre la de minimizar el tiempo y el esfuerzo implicados en las entrevistas, y hay que aplicar mucho ingenio a tales efectos. Por ejemplo, si los estudios se realizan en torno a personas que han sido afectadas por alguna comunicación radiofónica, se puede reducir el coste de tener que ir en busca de oyentes si se acude primero a los que escribieron a la emisora, refiriéndose a la comunicación. Cualquier sesgo en los resultados podrá evitarse si se conoce, por medio de los que escribieron a la emisora, a otras personas que escucharon el programa pero que no se sintieron obligadas a escribir acerca de él.

Los estudios psicológicos realmente intensivos podrían ser demasiado caros, si se llevan a cabo sólo para los fines de la investigación en la comunicación. Pero si se organiza bien la investigación, se podrían obtener resultados significativos si esa investigación específica se incluyera en otros estudios psicológicos e incluso psiquiátricos. Será posible obtener mucha información valiosa, con toda probabilidad, simplemente informando a los psicólogos y psiquiatras acerca de la importancia de la investigación en la comunicación. Unos resultados obtenidos de este modo son especialmente útiles, por cuanto que los estudios generales de los que formaron parte proporcionan una caracterización psicológica mucho más completa del entrevistado, en comparación con lo que normalmente se lograría.

Buena parte de esto es igualmente aplicable a la investigación mediante los estudios comunitarios: el coste adicional de la obtención de datos acerca de la comunicación y sus efectos en relación con un estudio comunitario resultaría ser relativamente bajo. De hecho, el estudio concreto de la pequeña ciudad del medio-oeste a la que se hacía referencia antes, aunque experimental en cuanto a sus características, supuso un gasto adicional de menos de 1.500 dólares, incluyendo el salario de un investigador formado al nivel actual de 3.000 dólares, los costes de alojamiento y desplazamientos, así como otros gastos incidentales en el campo. Ciertamente, pueden conseguirse los servicios de trabajadores plenamente competentes para conducir este tipo de investigación bajo su propia supervisión fácilmente por menos de 2.400 dólares por año.

Hasta la fecha, no existe ninguna medición del coste del análisis de contenido. En este caso, el principal gasto se destinará a los servicios de asistentes de investigación cualificados, pero la tasa anual media para este tipo de trabajo no habría de superar los 2.400 dólares. 
Claramente, es esencial una organización si tal investigación ha de ser viable económicamente: una organización que proporcione planificación, dirección y supervisión para la investigación, así como la coordinación de un proyecto determinado con otros proyectos relacionados. Una vez más, el ideal sería la integración completa en un instituto de investigación en la comunicación, comparable a los principales institutos de investigación en el campo de la economía. Existe ya para la radio una organización de esta naturaleza, la Oficina para la Investigación de la Radio de la Universidad de Columbia, que ya ha demostrado su capacidad de planificar y llevar a cabo proyectos de investigación especiales con relativamente poco gasto, conjuntamente con su principal proyecto. Lo mismo es aplicable, en gran medida, a la Escuela de Bibliotecarios de la Universidad de Chicago, que lleva años siendo un centro de investigación sobre la lectura. De hecho, el trabajo de estas dos organizaciones ya muestra las posibilidades de la coordinación, puesto que desde el inicio han colaborado en toda investigación que implicara una comparación entre leer y escuchar.

Lógicamente, se podría justificar, con toda probabilidad, una inversión en alguna organización para integrar toda la investigación en la comunicación. Si una organización tal no puede conseguir apoyo hasta que se haya demostrado aún más el valor de la investigación en la comunicación, no se podrán conseguir las economías y las ventajas que se derivarían de la integración. En este caso, el objetivo habrá de ser trabajar en pro de esa integración, en la medida de lo posible, a través de la colaboración informal entre quienes trabajan en este campo. 


\section{CRÍTICA DE LIBROS}

